\title{
Tense, Attitudes and Subjects
}

\author{
Alessandra Giorgi \\ University of Venice, Italy \\ giorgi@unive.it
}

\author{
Fabio Pianesi \\ IRST Trento, Italy \\ pianesi@irst.itc.it
}

\section{Introduction}

In this paper we address the nature of Sequence of Tense (SOT), and in particular the problem of its presence/absence in different contexts. We consider complement clauses, comparing the sentential complements of saying and believing (thinking, wishing, etc.) verbs, with those of fictional predicates, such as dream. The hypothesis we are going to argue for is that temporal anchoring is a requirement of certain contexts, not a property of verbal forms and tenses per se.

In order to implement this idea, we propose a view of the semantics of tenses in subordinate clauses based on the following tenets:

(i) the interpreted logical form (ILF) of clauses which are the object of propositional attitude predicates must represent the very attitudes episode; ${ }^{1}$

(ii) assignment sequences for the semantic interpretation can be subjectdependent, in that they can represent the temporal coordinate of the relevant subject (thinker, sayer, speaker, etc.) as the value of a distinguished variable, $x_{0}$;

(iii) tenses are subject-dependent indexicals, in that their interpretive contribution depends on the temporal coordinate of the relevant subject, as provided by the selected assignment sequence.

In short, propositional attitudes predicates (believe, wish, etc., but, crucially, not dream) are such that their objects must contain the coordinates of the subject of the attitude and in particular her temporal coordinate. By hypothesising that the temporal coordinate of a subject thinking, saying, etc. that $p$, is the episode itself, we will conclude that the object of a propositional attitude must explicitly represent the attitude episode. As a result, we will derive the fact that temporal anchoring is obligatory in contexts of propositional attitude (beliefs, wishes, etc.), whereas this is not the case in fictional ones.

This characterisation of temporal anchoring and SOT has important consequences for the theory of Double Access Readings (DAR). It will be argued that: a) the DAR actually involves double temporal anchoring of the embedded event, to both the matrix event and the utterance; b) this, in turn, involves an attitude by the speaker too, towards the content of the complement clause; and c) such an interpretive outcome relies on certain syntactic configurations in which the complementiser plays a major role.

This paper is organized as follows: in section 2 we consider the problem of why tenses don't have the same, mere indexical meaning in complement clauses as they have in matrix one. A first answer will be provided in section 3 , soon challenged by facts about dream contexts to be discussed in section 4 . 
Section 5 and 6 are devoted to presenting our theory, and its implementation in an ILF framework. Finally, in section 7, the theory is extended to the DAR.

\section{Temporal anchoring and propositional attitudes}

We start by considering the well-known problem of why tenses behave differently in complement clauses than in matrix one-that is, why there are no mere indexical readings of tenses in complement clauses? Or, to put it in a slightly different manner, 'why is sequence of tense obligatory?'. ' To exemplify, consider (1):
a. John said that Mary is sleeping.
b. Gianni ha detto che Maria dorme.

Utterances of both (1a) and its Italian counterpart, (1b), have only a double access reading. Roughly, in both cases the (sleeping) state depicted by the subordinate clause is perceived as holding both at the speaker's time and at John's/Gianni's. Now consider (2):

a. John said that Mary was sleeping.

b. Gianni ha detto che Maria dormiva(IMPF).

In both languages, the sleeping time is perceived as being either past (backwards shifted reading), or simultaneous to the sayer's. ${ }^{3}$

These phenomena are typical of many languages. Not of all, though; thus, in Russian the sentence corresponding to (1) yields a reading equivalent to the simultaneous one of (2). And the Russian counterpart of (2a) or (2b) only gives a backward shifted reading.

This crosslinguistic variations seem to exhaust the range of meanings of (1) and (2). Thus, there is no language in which the subordinate tense of (the counterpart of) (1) can be interpreted as a mere indexical, thence being suitable to report, on Saturday at five o'clock sharp, about the following dictum, as said by John on Friday:

(3) Tomorrow at five o'clock Mary will be sleeping.

Similarly, in no language the tenses of (2) can behave as mere indexicals-e.g., providing future oriented readings in which Mary's sleeping is constrained to be past only with respect to the speaker.

In the literature, it is possible to find suggestions that address at least some aspects of the problem we are considering. Thus, Ogihara (1996) and Higginbotham (1993) argue that the temporal orientation of clauses expressing the content of propositional attitudes must match that of the content they convey. Such a temporal isomorphism constraint can account for the non-availability of the intended reading of (2). That reading, in fact, would convey a future-oriented speech (as uttered by Gianni), by locating the relevant event in the speaker's past. 
But this would infringe the constraint, hence the reading is ruled out. Similarly, the lack of a mere indexical reading of the embedded present tense of (1) stems from the fact that the result would involve a present-time perspective by the utterer, and a future-time perspective by the subject (as in his/her original speech/attitude). As noted by Higginbotham (2001), the temporal isomorphism constraint has some problems, though. For instance, it requires some further working out to account for the acceptability of the following sentence (=Higginbotham's (23)):

(4) Maria will say on Sunday that Mario was here on Saturday.

Suppose (4) is uttered on Friday. Then the reported speech is past-oriented, from the standpoint of the subject (Mario), but future-oriented from the standpoint of the speaker. As such, it doesn't comply with temporal isomorphism, yet it is perfectly acceptable. Another possible, more conceptual, critique is that it is not clear why the contrasting perspectives of the speaker and the subject should matter.

Another relevant proposal can be found in Abusch (1997). Noticing the unavailability of the future-oriented reading of (2), she proposes that this is due a metaphysical (epistemic?) asymmetry between past and future times. The latter are intrinsically indeterminate, and this is reflected in a linguistic (interpretative) constraint to the effect that the local now, in Abusch's terminology, is an upper limit for tense reference (her Upper Limit Constraint).

Irrespective of the merits or limitations of these works, the main point is that their perspective is different from ours. They consider (1), (2) along with other examples that need be accounted for, in the course of discussing their approaches to the semantics of tense. On the other hand, we believe that the phenomenon these sentences exemplify-namely, the unavailability of mere indexical readings in complement clauses - is worth being given priority in the construction a theory of tenses. That is, we believe that whatever motivates the behaviour we've just discussed, that must be a fundamental property of tense, upon which a full theory is to be construed. Such a property arguably explains also the very existence of sequence of tense.

\section{A first attempt}

Let us start by making the rather uncontroversial hypothesis that tenses are relational devices that connect eventualities to temporal anchors. ${ }^{4}$ The observations in the previous sections suggest that tenses behave differently in complement clauses than in (ordinary) matrix ones. In the latter, they manifest an indexical behaviour, choosing the very utterance as their temporal anchor. In complement clauses tenses primarily worry about connecting the embedded eventuality with the matrix one. So, it seems that the following generalisation holds: 
a. The eventuality of an embedded clause is temporally anchored iff an explicit temporal relation between it and the eventuality of the embedding clause is available.

b. Temporal anchoring is obligatory for embedded clauses.

Such a generalisation both attempts at defining 'temporal anchoring', and stipulates the way it is expected to apply in embedded contexts. In an ILF framework (Higginbotham (1991); Larson and Ludlow (1993); Larson and Segal (1995)) (5a) can be taken to require the ILF of the embedded clause to include, as the value of the T node, a pair $\left\langle e, e^{\prime}\right\rangle$ consisting of the embedded eventuality, $e$, and the matrix one, $e^{\prime}$. This obviously predicts the facts observed above. Sentences (1a) and (1b) cannot have the readings we were discussing, because they would not comply with (5). The ILF for those readings, in fact, would feature a relation between the embedded event and the utterance, but nothing referring to the matrix event. Hence, the event of the subordinate clause would not be temporally anchored. Similarly, the sentences in (2) don't yield a future-oriented reading because that would correspond to an ILF in which the embedded eventuality is linked to the utterance, but not to the matrix events, this way, again, violating (5).

Thus, (5) seems to be capable to capture the general impossibility of embedded indexical tenses, thanks to the definition given for temporal anchoring, and by making it obligatory in embedded contexts. Mere indexicality is banned from embedded context because it results in non-anchored ILFs of the kind given in (6b). The possible readings, on the other hand, have the general form in (6a):

$$
\begin{array}{ll}
\text { a. } & {[\ldots \mathrm{T},\langle e, e\rangle \ldots . .]} \\
\text { b } & {[\ldots \mathrm{T},\langle e, u\rangle \ldots]}
\end{array}
$$

Promising as it is, this line of explanation collapses when confronted with facts concerning dream contexts, to which we now turn.

\section{Dreams}

In Giorgi and Pianesi (2001), we argued that dream contexts do not require the subordinated event to be temporally anchored. For instance, in (7) the dreamed event does neither precede nor overlap the matrix event (the dream itself), nor bear any particular temporal relation to the utterance:

a. Mario ha sognato che Carlo vinceva al totocalcio.

Mario dreamed that Carlo won(IMPF) the lottery.

b. John dreamed that Mary was sick.

On the contrary, utterances of both sentences are most naturally interpreted as presenting the winning as temporally un-related with respect to both the dream and the utterance. 
Further support to the idea that in sentences such as (7a) there is no temporal anchoring comes from the observation that the imperfect is a past tense, hence not compatible with future-oriented temporal phrases:

a. *Domani Maria partiva. Tomorrow, Maria left(IMPF).

b. *La settimana scorsa Gianni ha detto che ieri/oggi/domani Maria partiva.

Last week, Gianni said that yesterday/today/tomorrow Maria left(IMPF).

However, such a limitation disappears when the matrix predicate is sognare (dream):

(9) La settimana scorsa Gianni ha sognato che ieri/oggi/domani Maria partiva. Last week Gianni dreamed that yesterday/today/tomorrow Maria left(IMPF).

The lack of a connection between the embedded event and the dream can be given further support by showing that most of the phenomena normally associated with temporal anchoring are absent in dream contexts. Thus, in English a sentence like (10a) contrasts with (10b):

a. John dreamed that Mary ate a sandwich.

b. John said/believed that Mary ate a sandwich.

While in (10b) the eating cannot precede the saying/believing-i.e., it is backward shifted-the same does not happen in (10a). In other words, there is no sense in which the event of eating need precede that of dreaming.

\section{Towards a solution}

The data of the previous section, along with those discussed in the quoted work show that temporal anchoring is not enforced in dream contexts. ${ }^{5}$ Thus, the generalisation in (5) cannot be maintained as it is. In particular, (5b) must be amended, since it wrongly makes temporal anchoring a property of all complement clauses, irrespective of the matrix predicate. The data just discussed, however, point towards the opposite direction: temporal anchoring seems to be required by believe, say, fear, etc. but not by sognareldream. ${ }^{6}$

A fundamental difference between dream contexts and those enforcing temporal anchoring is that the former don't, whereas the latter do, entail a propositional attitude by the subject with respect to the content of the subordinate clause. To clarify this point, consider that utterances of sentences with matrix predicates such as say, believe, fear, etc. are understood as presenting the subject as either directly or indirectly having some kind of "concern" towards the truth of the embedded proposition. Thus, if John said/believed/feared that $p$, then he 
said(and-believed)/believed/feared that $p$ is true. This is clearly not the case with sognareldream. To say that John dreamed that $p$ doesn't entail that John dreamed that $p$ is true; the dream report, as such, is silent about whether there is any concern by the dreamer towards the truth of the dream.

We take such a concern with the truth of the propositional content to be a constitutive property of true propositional attitudes-namely, beliefs, fears, wishes, etc. For someone to be the subject of a true propositional attitude, it is not enough that there be a mental state/event of hers, with a given propositional content. It is also necessary that she care about/ be concerned with the truth of that content. In a sense, true propositional attitudes have a tripartite structure: they comprise a mental eventuality, an associated (propositional) content, and a concern by the subject towards the truth of that content. If this is correct, sognareldream is not a propositional attitude predicate: despite the fact it involves a mental eventuality and a content, it fails to entail any concern by the subject towards the truth of that content. ${ }^{78}$

Given this characterisation of propositional attitude, and of their differences with respect to dream contexts, it can be suggested that the presence/ absence of temporal anchoring depends on whether the relevant context is one of (true) propositional attitude, and modify (6b) accordingly. More precisely, we substitute (6) with (11):

a. Temporal anchoring amounts to the fact that the ILF of the embedded clause contains temporal relations between the event of the embedded clause, and that of the embedding one.

b. If a clause is the complement of a verb entailing a propositional attitude by the subject, then temporal anchoring obtains.

Such a move has an important conceptual consequence: temporal anchoring becomes the reflex of the expression of the concern (attitude) of a subject towards a propositional content. Empirical adequacy seems to be attained: say, in (1) and (2), is a predicate entailing a propositional attitude by the subject, hence temporal anchoring is obligatory and the mere indexical reading of the embedded clause is ruled out. At the same time, the facts concerning (7), which were problematic for (6), also follow: dream is not a predicate of propositional attitude, hence temporal anchoring is not enforced, and the event of the subordinate clause is free to float in an a-temporal realm.

We can simplify (11a), by removing the relational part and attributing it to the tense. After this move, temporal anchoring simply amounts to the requirement that the attitude eventuality be explicit represented in the ILF of the embedded clause. If we now merge this residue with (11b), we obtain (12):

(12) If a clause expresses the content of an attitude by a subject, then its ILF contains the attitude's eventuality.

Temporal anchoring, as usually conceived, has now disappeared. What remains is a condition on the form and content of the ILFs of clauses that are the objects of propositional attitude predicates, requiring that they contain the very attitude's episode. 
The general idea behind the present proposal is not foreign to a number of accounts. Thus, in the Reichenbachian (Reichenbach 1947) tradition of token reflexivity, Higginbotham's (1995) argues that by means of tense, thought contents make room for reference to their own episode (tensed thoughts). Similarly, though in a more indirect way, Stalnaker's (1984) diagonal construction is based on the claim that for there to be an attitude, the subject and the episode of her thought must exist in all possible worlds defining the attitude.

We understand these proposals, as well as (12), as focusing on the privileged role of subjects in propositional attitudes. Within an ILF framework, we take them to require that the ILF itself bear and appropriate mark of the subject, in order for it to express that subject's attitude. The appropriate mark is the subject's temporal egocentric coordinate-namely, the entity she uses to locate him/herself in time (Evans 1982)—which, in turn, is the attitude episode itself. Putting all this together, we have a (semi-)final formulation of what is now a condition on propositional attitude contexts:

\section{Conditions on propositional attitudes (first version):}

i) if a clause expresses the content of an attitude by a subject, then its ILF contains the subject's temporal egocentric coordinate;

ii) the primary role of tense is to locate the event with respect to such a coordinate;

iii) the temporal egocentric coordinate of the subject is the attitude's episode itself.

This condition preserves the suggested explanation for the impossibility of indexical readings of embedded tenses, while at the same time advancing our understanding of propositional attitudes. Indexical readings of tenses are unavailable in the object clauses of propositional attitude predicates because the corresponding ILF would contain the temporal coordinate of the wrong subjectnamely, the speaker, instead of the attitude's bearer. As before, the account still accommodates for dream contexts: they are not propositional attitude contexts, hence they do not fall under the strictures of (13).

As observed, temporal anchoring dissolves. It reduces to the (interface) requirement that a clause $C$ be 'marked' by the temporal coordinate of the right subject, in order for it to be usable to convey the content of a propositional attitude by that subject. ${ }^{9}$ Finally, the approach contributes a possible answer to the question of why do natural languages do have tenses at all, given that many other ways to temporally locate events (e.g., temporal phrases) are available. According to (13), the answer seems to be that what tenses contribute (their function) isn't simply a 'location' for events, but a way to appropriately mark propositional attitude contents according to the their bearers.

\section{Some details about ILF}

We now turn to sketching an ILF-based theory, which incorporates (13). To this 
end, we modify the ILF approach to propositional attitude contexts of Larson and Ludlow (1993) in two respects. In the first place, to make room for (13i) and (13iii), we propose that:

- Assignment sequences for attitudes are relativised to the subject's coordinate. For our purposes we distinguish between a speaker-oriented assignments, $\sigma_{s p}$, and a subject-oriented one, $\sigma_{s u b}$, where the subject is the bearer of the attitude.

- We are interested in values concerning the temporal coordinate of the subject, which we place at the 0 -th position of the sequence. Hence, for a propositional attitude context we will distinguish between $\sigma_{s u b}(0)$, the temporal coordinate of the attitude's subject, and $\sigma_{s p}(0)$, the temporal coordinate of the speaker. The former is assigned by the subject-oriented, and the latter by the speaker-oriented assignment sequence.

- $\sigma_{s u b}(0)$ is whatever value $\sigma_{s p}$ provides for the matrix event/state (the attitude episode). On the other hand, $\sigma_{s p}(0)$ will assign the 0 -th variable the utterance event. ${ }^{10}$

Concerning (13ii), we stick to our initial hypothesis that (at least certain) tenses are relational devices, which relate the (value of the) 0 -th variable to the (value of the) eventive one. Hence we can give the following lexical axioms for 'idealised' tenses:
a. $\operatorname{Val}(\langle a, e\rangle, \operatorname{Pres}, \sigma)$ iff $a \circ e$ and $\sigma(0)=a$
b. $\operatorname{Val}(\langle a, e\rangle$, Past, $\sigma)$ iff $e<a$, and $\sigma(0)=a$ etc.

Being sensitive to assignment sequences, the value of the distinguished 0 -th variable will vary according to whether evaluation is performed by using the speaker-oriented or the subject-oriented sequence. To exemplify, consider (15a).
a. John said that Mary was sick.
b. $[\ldots[\mathrm{T},\langle e, e\rangle \ldots]]$
$\sigma_{\text {sub }}$ is used, $\sigma_{\text {sub }}(0)=e^{\prime}$, and $e \circ e^{\prime}$
c. $[\ldots[\mathrm{T},\langle e, e\rangle \ldots]]$
$\sigma_{\text {sub }}$ is used, $\sigma_{\text {sub }}(0)=e^{\prime}$, and $e<e^{\prime}$
d. $*[\ldots[\mathrm{T},\langle e, u\rangle \ldots]]$
$\sigma_{s p}$ is used, $\sigma_{s p}(0)=u$, and...

Two possibilities are open for computing the ILF of the embedded clause: either by using $\sigma_{s u b}$, or by means of $\sigma_{s p}$. In the first case the 0 -th variable is assigned the same value the matrix eventive variable has been given by $\sigma_{s p}$, call it $e^{\prime}$. This possibility underlies both the simultaneous, (15b), and the backward-shifted reading, (15c). If $\sigma_{s p}$ is used, then we have (15d); an illegal ILF in view of (13), since it does not feature any reference to the attitude bearer's egocentric coordinate (the attitude episode). On the contrary, by referring to the utterance, it attributes the clause to the wrong subject.

Up to now, we have discussed and proposed a solution to the problem of why tenses behave differently in subordinate contexts than in matrix ones. We 
have argued that this is due to a general requirement about propositional attitude reports to the effect that the ILFs of their complement clause must feature a reference to the attitude subject's temporal coordinate. This restriction has been tied to a distinguishing property of propositional attitudes: for subject $\mathrm{X}$ to bear an attitude towards a propositional content it is necessary that $\mathrm{X}$ be concerned with the truth of that content. Only ILFs that bear the (right) subject's mark are amenable to function as a description of her attitude's content. Hence, the general ban against mere indexical readings in complement clauses is the effect of an interface condition aiming at securing that the ILFs expressing the content of propositional attitude be the right kind of objects. ${ }^{11}$

\section{A theory of the DAR}

It is everyone's intuition that in DAR sentences the embedded event is somehow connected both to the utterance and to the matrix event. Thus, and utterance of (16) is interpreted as suggesting that the pregnancy overlaps both the subject's and the speaker's time.

$$
\begin{aligned}
& \text { Gianni ha detto che Maria è incinta. } \\
& \text { Gianni said that Mary is pregnant. }
\end{aligned}
$$

In this section, we discuss a how the interface condition presented in the previous one can shed some light on the DAR phenomenon. We start by proposing to extend (13) by strengthening (13i) into a biconditional, this way yielding (17):

\section{(17) Conditions on propopostional attitudes (final version)}

(i) a clause is the object of an attitude by a subject iff the ILF of that clause contains the subject temporal egocentric coordinate;

(ii) the primary role of tense is to locate the event with respect to such a coordinate;

(iii) the temporal coordinate of the subject is the attitude's episode itself.

The hypothesis is that it is not only the case that the ILF of clauses expressing the content of an attitude contains the attitude's episode (the subject's temporal coordinate), but the converse holds as well: whenever the event of a clause is linked by tense to a subject's temporal coordinate, some attitude by that very subject must be at stake. Applied to (16), (17) suggests that the speaker has some kind of attitude of her own towards (part of) the content of the subordinate clause, so that the truth conditions are something along the following lines: ${ }^{12}$

$$
\begin{aligned}
& \exists e\left(\operatorname{say}(e) \wedge \operatorname{Agent}(e, \text { Gianni }) \wedge \operatorname{Theme}\left(e, / \exists e^{\prime}\left(\operatorname{pregnancy}\left(e^{\prime}\right) \wedge e^{\circ} e^{\prime} . . /\right)\right) \wedge\right. \\
& \exists e^{\prime \prime}\left(\phi\left(e^{\prime \prime}\right) \wedge \operatorname{Agent}\left(e^{\prime \prime}, \text { speaker }\right) \wedge \operatorname{theme}\left(e^{\prime \prime}, P\right)\right)
\end{aligned}
$$

That is, for an utterance $u$ of (16) to be true it must be the case that a) there is an event of saying, $e$, performed by Gianni, such that what is said is appropriately described by an ILF whose truth conditions are as shown by the material within 
backslashes in (18); and b) there is an attitude episode $e^{\prime \prime}$ of understood type $\phi$ whose object is the ILF $P$.

In other words, an utterance of (16) doesn't present the content of the subordinate clause only as (part of) the subject's attitude, but of the speaker's too. To make the proposal viable, we need investigate what $P$ consists of - namely, what is the content of the speaker's attitude in (16). We must also clarify how this can come about. Since the presence of the speaker's attitude is determined by the connection between the embedded event and the utterance, through the mediation of (17), and since such a connection is provided by the embedded tense, it must be shed light on how the tense can do the double work of linking the embedded event to both the matrix event and the utterance.

To understand what $P$ is, consider the following scenarios:

(19) Scenario: Mary is in the room.

John: Mary is in the room.

Scenario: Mary exits.

Speaker: \#John said that Mary is in the room.

Scenario: There is noone in the room.

John: Mary is in the room.

Speaker: John said that Mary is in the room.

(21) Scenario: There is no-one in the room.

John: Mary is in the room.

Scenario: Mary enters the room.

Speaker: \#John said that Mary is in the room.

The speaker's utterance in (19) is not appropriate, and the reason is not that the speaker has misrepresented John's thought/dictum. Rather, it seems to be traceable to the fact that she knows that things have changed since John said what he did, and have changed in respects that are relevant to the truth of John's utterance/thought. In particular, the speaker knows both that Maria was in the room when Gianni said what he said, and that she is no longer there, at the time of her own utterance. Eventually, the utterance is odd because the speaker has misrepresented the situation.

For similar reasons, even the speaker's utterance of (21) is not appropriate: she knows both that Mary was not in the room when John said what he said, and that she entered later. On the other hand, if Mary's staying in the room obtained at the subject's s and at the speaker's time, or at neither, then the speaker is entitled to say John said that Mary is in the room (cf. 20). 
So, it seems to be part of what the speaker conveys by using the presentunder-past sentences that at a certain point John talked as if Mary was in the room, and she holds that (in a sense) the situation has not changed since.

As a first approximation, we can attempt to capture the particular notion of a 'situation which has not changed in the meanwhile', by means of the following biconditional:

$$
\forall e[\text { in-the-room }(e) \wedge \text { theme }(e, \text { Maria })]\left(e^{\circ} e^{\prime} \leftrightarrow e^{\circ} u\right)
$$

where $e^{\prime}$ and $u$ are, respectively, the subject's and the speaker's temporal coordinates. With this, an utterance of John said that Mary is in the room is true iff: (i) Gianni said something to the effect that Mary was in the room at his temporal coordinate, and (ii) the speaker holds that every state of being-in-theroom involving Maria is such that it holds at Gianni's temporal coordinates iff it holds at the speaker's.

If the speaker holds something whose truth conditions are as exhibited by (22), then her utterance will be appropriate in the considered scenarios only in case Mary has not been in the room as far as the relevant period is concerned, or has continuously been there since John's saying. On the other hand, the cases in which Mary is in the room and then leaves, or is not in the room at John's time and then enters are excluded.

In our framework, in which ILFs and subject/speaker-oriented assignments play a major role, $(22)$ is a very rough way to accommodate the idea of a situation that has not changed (in relevant respects). A more precise, and more interesting way to account for the idea that a speaker uttering a DAR sentence holds that the situation has not changed in the meanwhile, consists in hypothesising that she holds that the two assignments (her and the subject's) are sufficiently similar to yield coordinated truth values. If the subject-oriented assignment is such that it makes the subordinate clause true, so does the speakeroriented assignment. On the other hand, if the clause is false under one assignment, then it is false under the other as well.

\subsection{Deriving the truth conditions for the DAR}

We turning now to the problem of how embedded tenses can do the double work of linking the embedded event to both the subject's and the speaker's temporal coordinate.

In Giorgi and Pianesi (1997) we proposed a split-C framework according to which two distinct complementisers can be singled out in embedded clauses: a lower one, called MOOD, related to the presence of an embedded subjunctive verb, and a higher one, C. In a successive work (Giorgi and Pianesi 2000) we argued in favour of a strict relationship between the DAR and the higher complementiser $\mathrm{C}$, to the effect that the $\mathrm{DAR}$ is possible iff $\mathrm{C}$ is projected (irrespective of the specific tense chosen). To explain this relationship, we hypothesised that the complementiser $\mathrm{C}$ is endowed with temporal features ( $\tau$-features) entering a relation with those of the embedded $\mathrm{T}$, by either movement or matching. As a result, when the ILF for the embedded clause of (16) is 
computed and the interpretive contribution of tenses is spelled out, the eventive variable is the same in $\mathrm{C}$ as in $\mathrm{T}$ :

$$
\begin{aligned}
& \text { a. }[\ldots .[\mathrm{v} \text { said }[\mathrm{CP}[\tau-\mathrm{C}[\mathrm{xP} \ldots \tau-\mathrm{T} \ldots]]]] \\
& \text { b. }\left[\ldots\left[\mathrm{C},\left\langle e, x_{\delta}\right\rangle \ldots .\left[\mathrm{T},\left\langle e, x_{\delta}\right\rangle \ldots . .\right]\right]\right]
\end{aligned}
$$

We now hypothesise that $\mathrm{C}$ is associated with $\sigma_{s p}$-that is, that the complementiser $\mathrm{C}$ is invariably associated with the speaker-oriented assignment sequence. ${ }^{13}$ Finally, we introduce the following axioms:

$$
\begin{aligned}
& \operatorname{Val}(\langle e, x\rangle,[\mathrm{V}[\mathrm{cP} C[\mathrm{XP} \ldots]]], \sigma) \text { iff for some } y, \operatorname{Val}(\langle e, x, y\rangle, \mathrm{V}, \sigma) \text { and } \\
& y=/ \mathrm{XP} /_{\sigma_{s u b}}
\end{aligned}
$$

$$
\operatorname{Val}(\mathrm{t},[\mathrm{cP} \tau-\mathrm{CXP}], \sigma) \text { iff for any } z, \operatorname{Val}(z,[\tau-\mathrm{C}], \sigma) \text { and } \operatorname{Val}\left(z, \mathrm{XP}, \sigma_{s u b}\right)
$$

Axiom (24), is used to compute the semantic value of phrases of the form $[V[C P$ $[X P \ldots]]]$, where $V$ is a verb of propositional attitude, and $[C P[X P \ldots]]$ is its complement. The axiom requires the ILF of the complement clause to be computed using the subject-oriented sequence, and skipping the $\mathrm{C}$ node. As a consequence, the $\mathrm{T}$ node of the embedded clause is assigned a pair consisting of the value for the embedded eventive variable, and the value for the matrix one, this way yielding ILFs of the following type: ${ }^{14}$

$$
\left[\mathrm{XP}, \mathrm{t} \ldots . .\left[\mathrm{T},\left\langle e, e^{\prime}\right\rangle \ldots\right]\right]
$$

As can be seen, this ILF accounts for the 'normal' subject-oriented, temporal anchoring; hence, it accounts for the attitude of the subject towards the content of the subordinate clause. Eventually, we can make sense of the fact that in order for an utterance of (16) to be true it must be the case that Gianni said something which is expressed by the ILF in (26), where the relevant event/action is presented as simultaneous to Gianni's (=the subject's) temporal coordinate.

Axiom (25) is meant to capture the intuition we discussed at the end of the previous section: by using a DAR sentence, the speaker commits him/herself to the idea that her assignment sequence, and the subject's are basically similar, in that they can provide for coordinate truth conditions. (25) implements this idea by assigning the whole CP headed by $\tau-\mathrm{C}$ the value $t$ under the speaker-oriented assignment iff the two constituents, the $\tau$-C node and its complement, have the same value truth value, the first under the speaker-oriented and the second according to the subject-oriented assignment.

It should be noticed that the ILF for XP is re-computed, according to (25). In particular, the variable $x_{0}$ of the embedded tense is re-assigned the value $\sigma_{s u b}(0)=e$; the variable $x_{0}$ in $\mathrm{C}$ is assigned $\sigma_{s p}(0)=u$; and the eventive variables associated with $C$ and $T$ receive the same value. As a result we have two ILFs: 

a. $\operatorname{ILF} 1=[\mathrm{C}, \mathrm{z}[\mathrm{C},\langle e, u\rangle \ldots]]$

b. $\operatorname{ILF} 2=\left[\mathrm{XP}, \mathrm{z}\left[\mathrm{T},\left\langle e, e^{\prime}\right\rangle \ldots\right]\right]$

$\tau$-C and $T$ have corresponding sets of $\tau$-features, hence express the same relationship. Because of this, ILF1 in (27a) is such that C is paired with value $z=t$ iff $e$ and $u$ (the speaker's temporal coordinate) are in the appropriate relation specified by the $\tau$-features, say $R$. As to ILF2, (27b) requires that $e$ be in the same relation $R$ to $e^{\prime}$, the subject's temporal coordinate. Finally, (25) requires that the whole $\mathrm{CP}$ be true just in case the two constituents, $[\tau-\mathrm{C}]$ and $[\mathrm{XP} .$.$] , have the same$ semantic value, this way providing for the connection between the truth conditions of the two ILFs. Applied to example (16), (25) delivers the required truth conditions: the two ILFs, in fact, have the same event (a pregnancy) constrained to be simultaneous to the speaker's temporal coordinate (27a) and to the subject's (27b). Moreover, they are required to have the same truth value. As a result, a pregnancy obtains at the subject's temporal coordinate iff it obtains at the speakers.

As noticed, axiom (25) requires computing the ILF of the XP clause. This might seem strange, in view of the fact that application of (24) is already capable of delivering such an ILF. There are empirical reasons, though, which justify maintaining (24) and (25) as they are-namely, with (25) independently requiring that the ILF for XP be computed by using $\sigma_{s u b}$. Those reasons come from the analysis of a phenomenon involving dream contexts.

In section 3 we saw that utterances of sentences such as (28) have readings in which the event mentioned in the embedded clause, the dreamed event, need not be temporally related to either the subject's temporal coordinate or the speaker's:

(28) Gianni ha sognato che Maria mangiava.

Gianni dreamed that Maria ate(IMPF).

It is a striking fact that when the tense in the subordinate clause is changed to a non-imperfect one, temporal anchoring is again available:

(29) La settimana scorsa Gianni ha sognato che ieri Maria vinceva /*ha vinto al totocalcio.

Last week Gianni dreamed that yesterday Maria won(IMPF/PAST)/*has won the lottery.

The sentence is fine with the imperfect, but not with the present perfect. The incompatibility of the present perfect with the future-oriented temporal phrase shows that with such a verbal form temporal anchoring is enforced. Thus, in (30a) the winning is perceived as past with respect to both the speaker and the dreamer, and in (30b) as in the future with respect to both: 
(30) a. Gianni ha sognato che Maria ha vinto al totocalcio.

Gianni dreamed that Maria has won the lottery.

b. Gianni ha sognato che Maria vincerà al totocalcio.

Gianni dreamed that Maria will win the lottery.

Classical double access readings are also available. Hence, (31) is similar to (16) in all relevant respects:

(31) Gianni ha sognato che Maria è incinta.

Gianni dreamed that Maria is pregnant.

These contexts, which Giorgi and Pianesi (2001) have dubbed evidential dreams, have many other interesting peculiarities. We won't discuss them here, being enough for our purposes to draw attention to the fact that the use of non-imperfect tenses in dream contexts brings temporal anchoring back on the stage.

Now, if our characterisation of sognare/dreams is correct, their not being verbs of propositional attitude is not a contingent properties of theirs. That is, it is not limited to the particular tense arrangements we saw in section 3 . On the contrary, it seems to be a defining property of sognare/dream that dreamers are not concerned with the propositional contents of dreams, much as it is a defining property of beliefs or wishes that believers/wishers do care about the truth of the relevant contents. Thus axiom (24) is no more appropriate for sentences such as (30) than it was for (28). When the main verb is sognare/dream, what is needed is an axiom like (32):

$$
\begin{aligned}
& \operatorname{Val}(\langle e, x\rangle,[\mathrm{V}[\mathrm{CP} \mathrm{C}[\mathrm{XP} \ldots]]], \sigma) \text { iff for some } y, \operatorname{Val}(\langle e, x, y\rangle, \mathrm{V}, \sigma) \text { and } \\
& y=/\left.\mathrm{XP}\right|_{\sigma^{*}}
\end{aligned}
$$

where $\sigma^{*}$ is an assignment sequence that, differently than $\sigma_{s u b}$ and $\sigma_{s p}$, does assign the 0-th variable neither the speaker's nor the dreamer temporal coordinate. ${ }^{15}$ The result, of course, is an ILF for the subordinate clause, which does not exhibit temporal anchoring. Putting all together, we obtain that the truth of both (28) and (29) requires that Mario dreamed something that is expressed by the relevant, non-anchored ILF. But, then, how can the temporal sensitivity of (29) - namely, the fact that in (29) the dreamed event must precede both the utterance and the dream-be explained?

It is at this juncture that (25) has an important role to play. Temporal anchoring in (29)-(31) is primarily towards the speaker, hence expected to be controlled by the same axiom that, in the general case of DAR, accounts for the speaker oriented portion of meaning. What is needed, for (25) to come into play, is that the LF structure of (29)-(31) be the same as that of (16), an axiom ruling the interpretive behaviour of clauses headed by a $\mathrm{C}$ endowed with $\tau$-features. This seems to be the case, as argued in Giorgi and Pianesi (2001).

Our hypothesis, then, is that the temporal sensitivity of (29)-(31) is a side effect of (25): Among the things that axiom does there is the requirement that the XP clause be assigned an ILF computed with respect to $\sigma_{s u b}$. Hence, (25) by itself 
suffices, and, as it turns out, is necessary to account for the facts we've just reviewed. The desired result is then obtained: It is a component of the meaning of an utterance of (31) that the speaker holds that for any state of the relevant kind, such a state overlaps her own temporal coordinates iff it does the so with the dreamer's ones. ${ }^{16}$

Conceptually, the conclusion is quite important. The temporal orientation towards the subject in (30) is secondary to (25). The latter, in turn, and the DAR tout court, depend on a specific syntactic configuration, in which the higher complementiser has $\tau$-features entering in a relation with those of the embedded $\mathrm{T}$.

\section{Conclusions and future work}

In this work we have been mainly concerned with foundational issues about sequence of tense. The problem was why languages need SOT at all, instead of resorting to what seems the most straightforward strategy-namely, allowing tenses to behave in complement clauses the same way they do in matrix ones.

By comparing propositional attitude contexts with dream ones, we argued that SOT is the (linguistic) expression of a constitutive property of propositional attitude contexts: The representation expressing their contents need to make room for the temporal coordinates of the attitude bearer, in the form of the very attitude episode. The securing of these interface conditions is performed by a semantic machinery relying on interpreted logical forms as the representational means of attitude contents, and on assignment sequences which are sensitive to attitude bearers, this way incorporating their perspectives.

We believe that this theory opens a number of interesting research paths. Two of them, which have only been mentioned in the course of the discussion, deserve further investigation. The first concerns the role of tenses in language. As already noticed, if our theory is correct, the possibility arises that their role is not that of locating eventualities, but that of satisfying abstracts requirements on the form and contents of the ILFs to the effect that they be marked by the appropriate attitude bearer.

The second, more speculative, line of investigation refers to a conception of the mind as consisting of interacting modules, among which the language module and that devoted to processing representations concerning other people's mental states can be found. It is then possible to hypothesise that the latter, the Theory of Mind (TOM) module works on representations delivered by the former in the form of ILFs (Segal 1998). If so, the constraints we have been concerned with in this paper can be related to the way the TOM module requires its input to be formatted. For instance, the subject's mark (the attitude's eventuality) on ILFs would be needed to keep the mental representation of other people's mind mutually distinguishable, allowing the relevant processes to operate selectively on them.

On a less speculative path, the kind of theory of SOT that is emerging seems to provide valuable insights on many phenomena. In this work we discussed an application of these ideas to the phenomenon of DAR. In our view, 
the DAR is a sign of a direct concern of the speaker with respect to the content expressed by the subordinate clause-a possibility which we've accounted for by strengthening the relationship between temporal anchoring and attitude contexts into a biconditional. At the same time, the DAR relies on a certain syntactic configuration whereby the higher complementiser, $\mathrm{C}$, plays a crucial role.

\section{Endnotes}

${ }^{1}$ For the notion of Interpreted Logical Form, see Higginbotham (1991), Larson and Ludlow (1993), Larson and Segal (1995).

${ }^{2}$ This is the title of a paper by J. Higginbotham (2001), where it is argued that the solution can be found by seriously considering the possibility that tenses (in subordinate contexts) are anaphoric.

${ }^{3}$ Actually, as we have shown at length in Giorgi and Pianesi (1997; 2000), the Italian example admits a backword shifted reading only when the contexts makes available a suitable temporal/eventive entity. Indeed, such an entity provides a referent for the anaphoric imperfect tense found in (2b). For the time being, however, we ignore these peculiarities and treat $(2 a)$ and $(2 b)$ on a par.

4 Well, not so uncontroversial, actually, for there are other views about the semantics of tenses: as more-or-less- refential terms, as operators, etc. Let's say that the hypothesis in the text is meant to not prejudice extension of what we will say to other approaches.

${ }^{5}$ Notice, they do not show that that it is impossible to construe mutual temporal orientation for events internal to dream reports.

(i) Mario ha sognato che sua madre mangiava. Era appena ritornata dal lavoro.

Mario dreamed that his mother ate(IMPF). She had(IMPF) just returned from work.

Here the mother's return from work is clearly past with respect to her eating. However, both are unordered with respect to the matrix event-namely, the dream.

${ }^{6}$ Incidentally, phenomena similar to those of section 4 are found in statements describing the content of fictions:

(i) In Moby Dick, Achab riesce/riusciva ad uccidere la balena bianca. In Moby Dick, Achab manages/managed(IMPF) to kill the white whale.

Clearly, the killing of the whale, the fictional event, is not located with respect to the utterance, a fact stressed by the possibility of using both the imperfect and the present tense in these contexts.

This could suggest a line of explanation according to which the absence of temporal anchoring (=the normal behaviour of tenses) is somehow motivated by the fact that statements such as (i), and dream contexts report about non-actual situations. However, this strategy risks to neglect the fact that temporal anchoring 
is available in reports about beliefs, wishes, etc. which also involve non-actual situations concerning what is believed, wished, etc. Anticipating the theory to be developed, what seems relevant is not whether the situation is simply actual, but whether there is any given subject for which actuality is important.

${ }^{7}$ In the language of possible world semantics, it could be said that a sentence with main predicate wish presents the wisher as wishing that the actual world, the one she lives in, comply with the content of the proposition expressed by the subordinate clause. Similarly, a subject believes that $p$ iff she locates him/herself in a world where $p$ is true, believing that that world is the actual world. Thus, propositional attitudes involve the subject, the actual world and the relevant proposition, in such a way that: a) the subject contemplates the possibility that the proposition is true in the actual world, and b) she either holds it (belief), or gets involved in further emotional labour (fear, wish). It seems to us that the (a) point-the concern of the subjects towards the truth of the propositional content-qualifies propositional attitudes.

Dreams are different. For a sentence such as $X$ dreamed that $p$ to be true it need not be the case that $X$ dreams that the actual world is such that $p$ is true in it. One might insist that dream sentences are true iff the dreamer dreams to be in a world where the given state of affairs obtains. This is questionable (at least in the general case). However, even if this is accepted, it seems wrong to maintain that for those sentences to be true the dreamer needs to contemplate the truth of the proposition in the actual world. In this sense, the object of a dream does not necessarily involve the (actual) world, contrary to the object of propositional attitudes.

${ }^{8}$ Of course, these considerations can be extended to fictional statements, this way accounting for the observations in fn. 6.The situations which beliefs, wishes, etc. are about are actual situations for the subject of the attitude.

${ }^{9}$ One might venture that there's something irreducibly de-dicto in the embedded clause of a propositional attitude predicate-namely, the temporal coordinate of the subject, and, indirectly, the subject herself. At the same time, it is important to notice that (13) does not rule out the possibility that the embedded clause features an independent reference to the utterance, as in John said that Mary will be here tomorrow - that is, it does not rule out de-re (speaker-oriented) reference through such idnexicals as tomorrow, yesterday, etc. What (13) requires is that the needed de-dicto element be represented, and that this be obtained through the tense.

${ }^{10} \mathrm{At}$ least for our purposes, we take $\sigma_{\text {sub }}$ to be like $\sigma_{\mathrm{sp}}$ except for the fact that the former assigns the matrix event as the value of the 0-th variable., whereas the latter assigns the utterance.

What presented in the text can be given a more precise form by resorting to the notion of selection relation. In (Larson and Segal 1995), a selection relation, $\Sigma$, relates utterances (of a sentence) to assignment sequences, to the effect that only if it holds between utterance $u$ and assignment $\sigma, \Sigma(u, \sigma)$, the latter is appropriate for the former. In our case, we can modify the definition of selection relation to extend to triples consisting of an attitude episode, a clause and an assignment 
sequence. With this, a sequence $\sigma$ is appropriate for clause $C$ in the context of attitude $e$ iff $\Sigma(\sigma, C, e)$. We won't purse such a course any further here.

11 Following suggestions in the literature-notably Segal (1998)-it can be hypothesised that ILFs are fed into the Theory of Mind module. In a speculative mood, the kind of restrictions on ILFs we arrived at, could then be seen as due to requirements coming from such a module: in order for a representation to be processable as pertaining to X's mental contents, it must be appropriately marked by $\mathrm{X}$ itself.

${ }^{12}$ There is a good amount of literature on DAR. Of particular relevance are the works by Ogihara (1996), Abusch (1997), who propose a de-re theory. For reason of space, it is not possible to discuss their works in details, nor discuss what we take to be serious empirical and conceptual limitations of theirs. Our approach is more similar, at least in spirit, to Higginbotham (2001); see also (Giorgi and Pianesi 2000).

${ }^{13}$ As discussed in (Giorgi and Pianesi 2000), this move is justified by the fact that, independently of the level of embedding, the DAR is always and uniquely related to the event of the immediately embedding clause and to the utterance:

(i) Gianni ha detto che Mario ha affermato che Maria è incinta. Gianni said that Mario stated that Maria is pregnant.

In this sentence the pregnancy is perceived to be simultaneous to the temporal coordinates of Mario and of the speaker.

${ }^{14}$ In the lights of the considerations in fn.10, it would be enough that (24) require the ILF of the subordinate clause to be computed with respect to an assignment which is appropriate for the clause according to the selection relation:

(i) $\operatorname{Val}(\langle e, x\rangle,[\mathrm{V}[\mathrm{CP}[\mathrm{xP} \ldots]]], \sigma)$ iff for some $y, \operatorname{Val}(\langle e, x, y\rangle, \mathrm{V}, \sigma)$ and $y=/ \mathrm{XP} / \sigma$ where $\Sigma(\sigma,[\mathrm{XP} \ldots], e)$.

In general, it seems that mention of the assignment sequence can be omitted, provided that we understand that semantic values are relativised to appropriate assignment sequences.

${ }_{15} \mathrm{We}$ are purposely vague in specifying the characteristics of assignment sequences for dream contexts. All we need to require here is that they differ from the ones typical of propositional attitude contexts.

${ }^{16}$ This raises a problem with respect to (16), according to which as soon as an ILF is marked with the temporal coordinates of a given subject, that ILF expresses the content of an attitude by that subject.

At this point, the problem is empirical, and it is not clear to us whether attitudes of any kind by the dreamer towards the dream's content are entailed by, say, (30). At present, some data briefly discussed in Giorgi and Pianesi (2001) seem to point towards an answer in the affirmative. Obviously, should it turn out that this is not the case, (16) should be dropped, and we'd have to return to the weaker (13).

Whatever the conclusion of that investigation, however, it seems it's not going to hamper the hypothesis made in the text concerning the predicate sognare (dream). For, even if we were to embrace the thesis that in (30) there is a propositional 
attitude by the dreamer towards the dream's content, it would still be true that such an attitude is not the dream, hence does not depend on the verb sognare. As it seems plausible, that attitude of the dreamer towards the content of her own dream would be something external to the dream, possibly arising after it. It would not be specified linguistically, but through the context.

\section{Bibliography}

Abusch, D., (1997), "Sequence of Tense and Temporal De Re". In Linguistics and Philosophy, 20: 1-50.

Evans, G., 1982. The Varieties of Reference. Oxford University Press

Giorgi, A. and F. Pianesi, 1997. Tense and Aspect: from Semantics to Morphosyntax. Oxford University Press, New York.

Giorgi, A. and F. Pianesi, 2000. "Sequence of Tense Phenomena in Italian: a Morphosyntactic Analysis". In Probus, 12, 1-32.

Giorgi, A. and F. Pianesi, 2001. "Imperfect Dreams. The temporal dependencies of fictional predicates". In Probus, 13, 31-68

Higginbotham, J., 1991. "Belief and Logical Form". In Mind and Language, 6: 344369.

Higginbotham, J., 1995. “Tensed Thoughts”. In Mind and Language.

Higginbotham, J., 2001. "Why is Sequence of Tense Obligatory?”. In G. Preyer and E. Lepore (eds.) On Logical Form. Oxford University Press. New York.

Larson, R. and P. Ludlow, 1993. 'Interpreted Logical Forms'. In Synthese 95:305355.

Larson, R. and G. Segal, 1995. Knowledge of Meaning. The MIT Press, Cambridge MA.

Ogihara, T., (1996). Tense, Attitudes and Scope. Kluwer Academic Press, Dordrecht..

Reichenbach, H., 1947. Elements of Symbolic Logic. Mac Millan, New York.

Segal, G., 1998. 'Representing Representations'. In P. Carruthers and J. Boucher (eds.), Language and thought. Cambridge University Press.

Stalnaker, R.C., 1984. Inquiry. The MIT Press, Cambridge CA.

Stowell, T., 1996. 'The Phrase Structure of Tense'. In J. Roorick and L. Zaring (eds.), Phrase Structure and the Lexicon, 277-291. Kluwer Academic Publishers, Dordrecht. 